\title{
THE IMPORTANCE OF THE LAW OF THE WALL
}

\author{
B.C. MANDAL ${ }^{*}$ \\ Civil Engineering Department \\ Jalpaiguri Govt. Engineering College \\ Jalpaiguri- 735102, West Bengal, INDIA \\ E-mail: bcmjgec@gmail.com \\ H.P. MAZUMDAR \\ Physics and Applied Mathematics Unit \\ Indian Statistical Institute \\ Kolkata-700108, INDIA
}

\begin{abstract}
This paper reports some results of turbulent boundary layer computation. The calculation is made assuming that law of the wall is valid throughout the boundary layer. Simple relations are proposed for friction for a smooth pipe and a flat plate at zero incidence. The results are compared with recent measurements. Encouraging results are obtained for both the cases of flows.
\end{abstract}

Key words: boundary layer, flat plate, friction factor, skin friction.

\section{Introduction}

Major efforts have been made, theoretically or empirically, to relate Reynolds' stresses and the mean turbulent flow field but so far the result is not very satisfactory. In this paper, it is shown that the simple law of the wall can be used as the only input to solve some problems of turbulent boundary layers. Different flows of practical importance are considered for this purpose.

Prandtl (1933) concluded that the time mean velocity $u$ near the smooth wall must depend upon density $\rho$ and viscosity $\mu$ of the fluid, the shear stress at the wall $\tau_{w}$ and on the distance from the wall $y$. Thus, near the smooth wall there is a functional relationship

$$
u=u\left(\rho, \mu, \tau_{w}, y\right) .
$$

From dimensional analysis the functional relationship can be written in the from

$$
u / v_{*}=f\left(y v_{*} / v\right) .
$$

In which the shear velocity $v_{*}=\sqrt{\tau_{w} / \rho}$ and $v=\mu / \rho$. Introducing $u^{+}=u / v_{*}$ and $y^{+}=y v_{*} / v$, Eq.(1.2) can be written as

$$
u^{+}=f\left(y^{+}\right)
$$

\footnotetext{
* To whom correspondence should be addressed
} 
Equation (1.3) is called the law of the wall. Most widely used law of the wall is the logarithmic law which has the form

$$
u^{+}=\frac{1}{\kappa} \ln \left(y^{+}\right)+B
$$

where $\kappa$ is the von Karman constant and $B$ is the constant of integration. The logarithmic law (1.4) does not satisfy the no-slip condition at the wall and the condition of zero-slope of velocity profile at the outer edge of the boundary layer. No-slip condition can be satisfied if Spalding's (1961) formulation for the law of the wall is used. Spalding (1961) has given a special form of Eq.(1.3)

$$
y^{+}=f\left(u^{+}\right)=u^{+}+A\left[e^{\kappa u^{+}}-1-\kappa u^{+}-\frac{\left(\kappa u^{+}\right)^{2}}{2}-\frac{\left(\kappa u^{+}\right)^{3}}{6}-\frac{\left(\kappa u^{+}\right)^{4}}{24}\right]
$$

where

$$
A=e^{-\kappa B}=0.1108, \quad \kappa=0.4, \quad B=5.5
$$

Though no-slip condition is satisfied by Eq.(1.5), the condition of zero-slope of velocity profile at the outer edge of the boundary layer is not fulfilled.

The unique feature of Spalding's equation is that it presents $y^{+}$as a function of $u^{+}$rather than $u^{+}$as a function of $y^{+}$. These features have made computations using this law of the wall much easier to perform. The subsequent calculations will be based on this expression, but the development of the method is independent of the specific function $f\left(u^{+}\right)$. The aim of the present paper is to establish some relationship between flows resistance and the mean values of velocity components.

Figure 1 shows how Eq.(1.5) traces turbulent boundary layer data and pipe flow data. It can be seen easily that Spalding's formulation captures the viscous sub-layer and the so called overlap layer well without however describing the good trace of data points in the wake region. It is also observed that Spalding's (1961) law of the wall predicts pipe flow data better than boundary layer data.

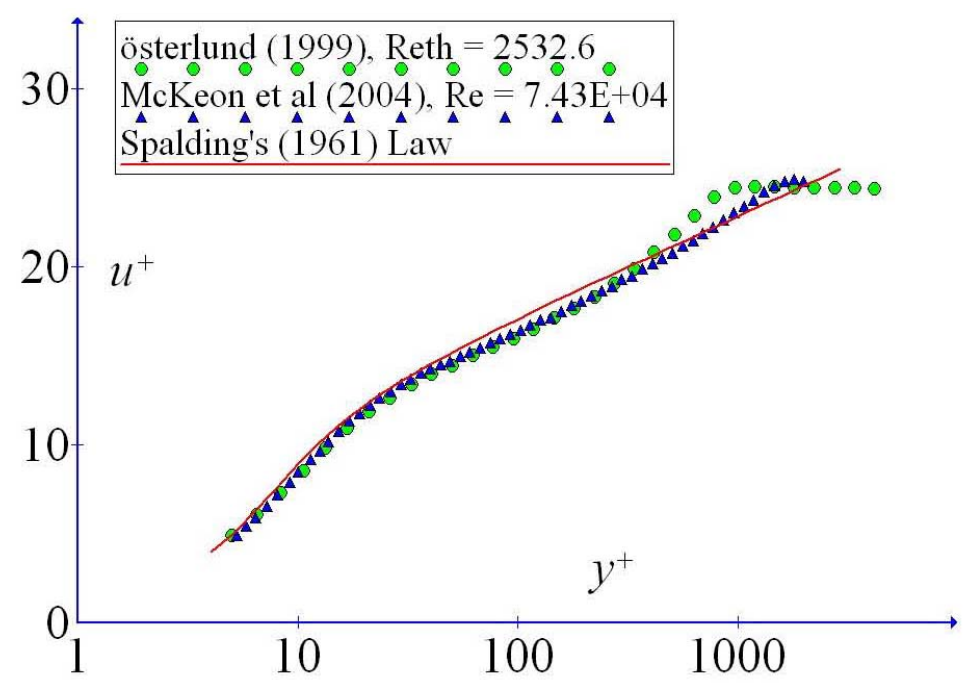

Fig.1. Spalding's (1961) law compared with the pipe flow and flat plate data. 


\section{Fully developed turbulent pipe flow}

The Reynolds number, Re, for the pipe flow is defined as

$$
\operatorname{Re}=\frac{V D_{H}}{v}
$$

where the hydraulic diameter $D_{H}=4 R_{H}$, the hydraulic radius $R_{H}=A / P, A$ is the cross sectional area and $P$ is the wetted perimeter. For the case of pipe flow $D_{H}=2 R$ where $R$ is the radius of pipe, then

$$
\operatorname{Re}=\frac{2 V R}{v} .
$$

The average velocity $V$ through the pipe may be defined in terms of the radial co-ordinate $r$ or wall co-ordinate $y$, where $y=R-r$ and $d y=-d r$. The distance $y$ is measured from the wall while $r$ is measured from the center of the pipe

$$
V=\frac{1}{\pi R^{2}} \int_{0}^{R} 2 \pi r u d r=\frac{2}{R} \int_{0}^{R} u d u-\frac{2}{R^{2}} \int_{0}^{R} u y d y .
$$

Introducing $u^{+}=u / v_{*}, y^{+}=y v_{*} / v$ and $R^{+}=R v_{*} / v$ in Eq.(2.3), it becomes

$$
\operatorname{Re}=\frac{2 V R}{v}=4 \int_{0}^{R^{+}} u^{+} d y^{+}-\frac{4}{R^{+}} \int_{0}^{R^{+}} u^{+} y^{+} d y^{+}
$$

where $R^{+}=f(\xi)$ is the value of $y^{+}$when $u^{+}=\xi=U_{c} / v_{*}$ in which $U_{c}$ is the velocity on the pipe centerline.

Using Spalding's formulation the first integral of Eq.(2.4) becomes

$$
\int_{0}^{R^{+}} u^{+} d y^{+}=\frac{\xi^{2}}{2}+\frac{A}{\kappa}\left[e^{z}(z-1)+1-\frac{z^{2}}{2}-\frac{z^{3}}{3}-\frac{z^{4}}{8}-\frac{z^{5}}{30}\right]
$$

where $z=\kappa \xi$. The second integral of Eq.(2.4) will be very cumbersome. It is computed numerically. The computed values of two integrals are shown in Fig.2.

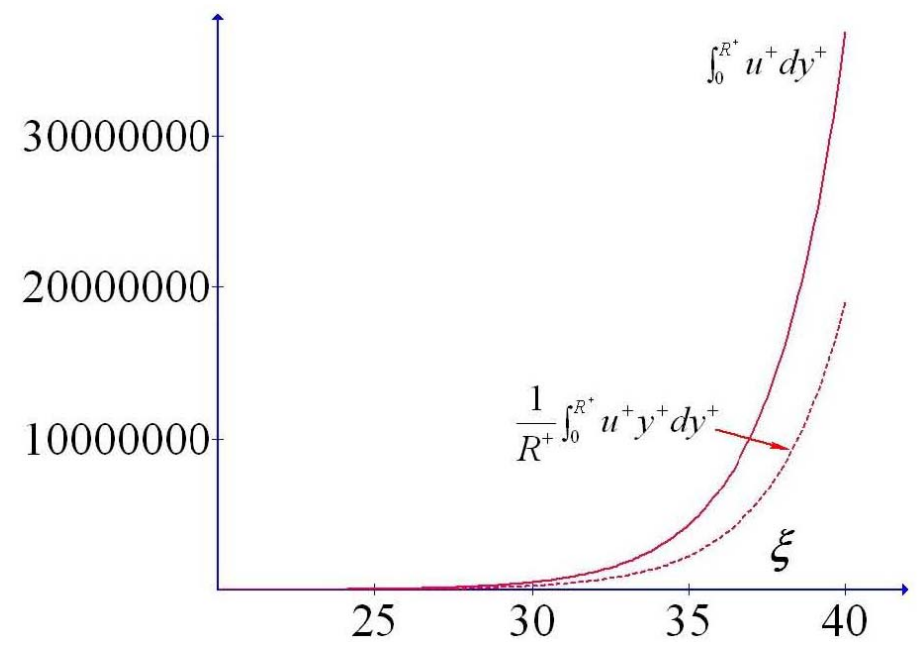

Fig.2. Variation of two integrals. 
The Darcy-Weisbach friction factor for a smooth pipe can be written as

$$
\begin{aligned}
& f=\frac{8 g R_{H} S}{V^{2}}=8 \frac{v_{*}^{2}}{V^{2}}=4 \frac{\rho v_{*}^{2}}{\frac{1}{2} \rho V^{2}}=4 \frac{\tau_{w}}{\frac{1}{2} \rho V^{2}}=4 C_{f}, \\
& f=8 \frac{v_{*}^{2}}{V^{2}}=32 \frac{R^{2} v_{*}^{2} / v^{2}}{4 V^{2} R^{2} / v^{2}}=32 \frac{\left(R v_{*} / v\right)^{2}}{(2 V R / v)^{2}}=32\left[\frac{R^{+}}{\mathrm{Re}}\right]^{2}
\end{aligned}
$$

where $g$ is the gravitational constant for acceleration, $S$ is the slope, shear velocity $v_{*}=\sqrt{g R_{H} S}=\sqrt{\tau_{w} / \rho}$, $\tau_{w}$ is the shear stress at the wall and $C_{f}$ is the coefficient of skin friction.

For a value of $\xi, R^{+}=f(\xi)$ can be calculated from Spalding's formulation, and then Re can be calculated from Eqs (2.4), (2.5) and Fig.2 and finally the friction factor $f$ can be estimated from Eq.(2.6). The computed values are compared with the measured data of Swanson et al. (2002) and McKeon et al. (2004) in Fig.3.

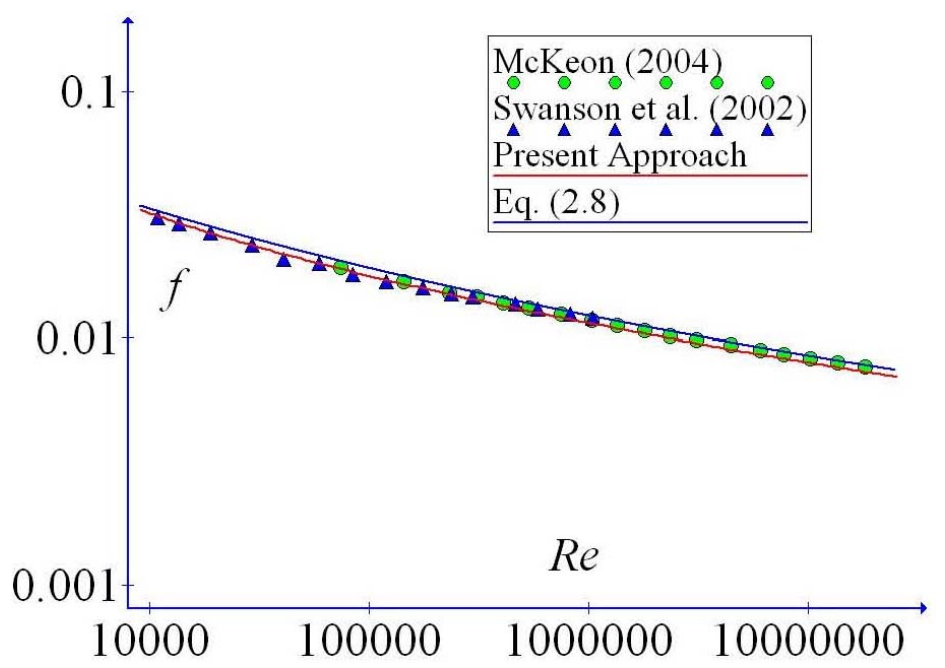

Fig.3. Computed friction factor compared with experimental data. determined

Substituting the logarithmic law in Eq.(2.3) the average velocity $V$ through the pipe can be

$$
V=v_{*}\left(\frac{1}{\kappa} \ln R^{+}+B-\frac{3}{2 \kappa}\right)
$$
obtained

Combining Eq.(2.7) and Eq.(2.6) the relation between the Reynolds number and friction factor is

$$
\frac{1}{\sqrt{f}}=1.99 \log (\operatorname{Re} \sqrt{f})-1.02
$$


where constants $\kappa=0.41$ and $B=5$ are considered. This formula was derived by Prandtl in 1935 . Equation (2.8) is also plotted in Fig.3. It is observed that Eq.(2.8) differs very much from experimental data. This difference may be attributed to the fact that the logarithmic law is not a good approximation for the sub-layer and wake region.

Prandtl (1935) adjusted the constants of Eq.(2.8) using the experimental data of his student Nikuradse (1932). The adjusted equation is given below

$$
\frac{1}{\sqrt{f}}=2.0 \log (\operatorname{Re} \sqrt{f})-0.8 \text {. }
$$

This is Prandtl's (1935) law of friction for a smooth pipe. The friction factor obtained in the present approach is compared with Prandtl's (1935) law of friction in Fig.4. It is revealed that the friction factor computed from the present approach is very much close to Prandtl's adjusted equation.

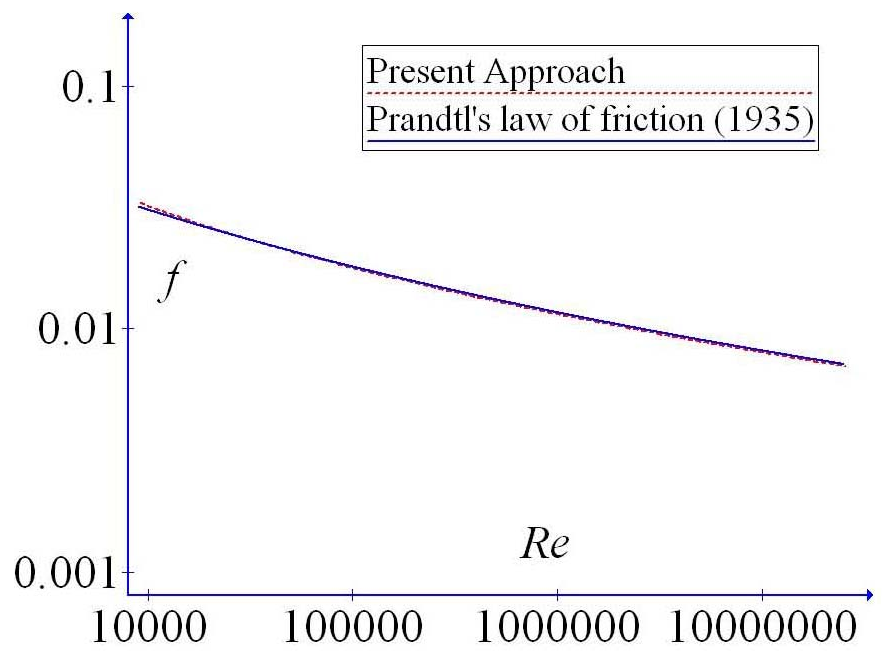

Fig.4. Comparison between two theoretical $f(\mathrm{Re})$ relations.

Shear stress distribution for fully developed pipe flow

$$
\frac{\tau}{\tau_{w}}=1-\frac{y^{+}}{R^{+}}=1-\frac{f\left(u^{+}\right)}{f(\xi)}
$$

where $\tau$ is the shear stress at any distance $y$ from the wall and $\tau_{w}$ is the shear stress at the wall.

One may subtract the viscous portion of the shear tress $\tau_{l}$ from it whereby one is left with the "turbulent" shear stress $\tau_{t}$. Thus

$$
\tau_{t}=\tau-\tau_{l}
$$

where

$$
\tau_{l}=\mu \frac{d u}{d y}=\tau_{w} \frac{d u^{+}}{d y^{+}}=\tau_{w} \frac{1}{f^{\prime}\left(u^{+}\right)}
$$


and consequently

$$
\frac{\tau_{t}}{\tau_{w}}=1-\frac{f\left(u^{+}\right)}{f(\xi)}-\frac{1}{f^{\prime}\left(u^{+}\right)}
$$

$\tau_{t} / \tau_{w}$ has been computed from Eq.(2.12) using Spalding's formulation and the results are compared with Laufer's (1954) data in Fig.5. The results are in good agreement with the experimental data.

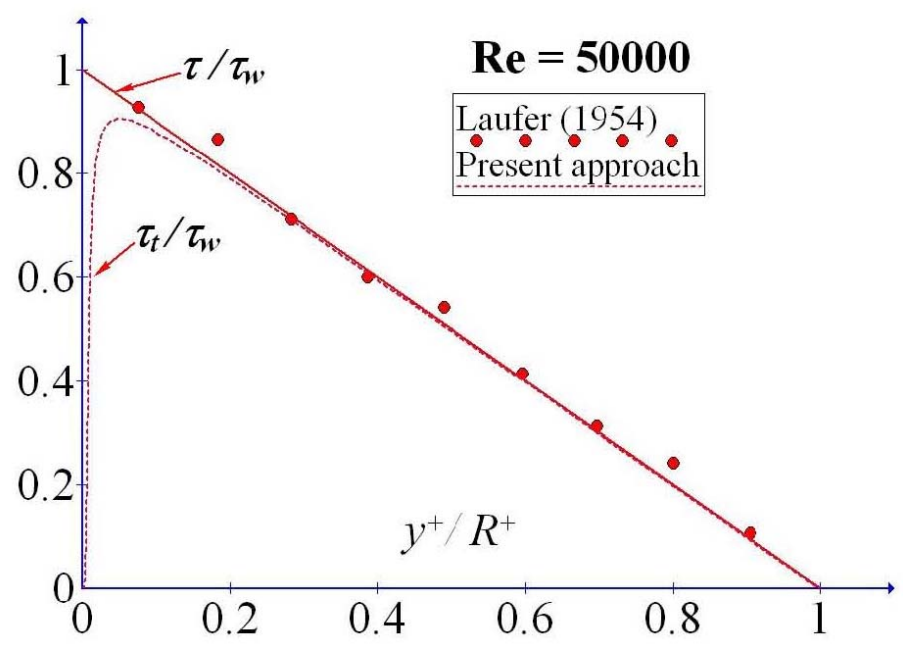

Fig.5. Computed shear stress compared with Laufer (1954).

\section{Turbulent flow over a flat plate}

Kestin and Persen (1962) deduced the momentum equation for a flow over a smooth flat plate in a zero pressure gradient flow

$$
\frac{1}{\rho} \frac{\partial \tau}{\partial y}=\frac{\nu_{*}}{\mu} \frac{\partial \tau}{\partial y^{+}}=v_{*} \frac{d v_{*}}{d x} u^{+2}
$$

where $x$ is the downstream distance parallel to the plate. It was assumed $u^{+}=u(x, y) / v_{*}(x)=f\left(y^{+}\right)$only.

Upon integrating Eq.(3.1) with respect to $y^{+}$, the shear stress distribution across the boundary layer is obtained

$$
\tau-\tau_{w}=\mu \frac{d \nu_{*}}{d x} \int_{0}^{y^{+}}\left(u^{+}\right)^{2} d y^{+}=\mu \frac{d \nu_{*}}{d x} G\left(u^{+}\right)
$$

where

$$
G\left(u^{+}\right)=\int_{0}^{y^{+}}\left(u^{+}\right)^{2} d y^{+}
$$

At the edge of the boundary layer 


$$
\tau \rightarrow 0 \quad \text { as } \quad{ }^{+} \rightarrow \delta v_{*} / v=y_{o}^{+}, \quad u^{+} \rightarrow U_{\infty} / v_{*}=\xi
$$

where $y_{o}{ }^{+}$is the dimensionless boundary layer thickness, $\xi$ is the dimensionless free stream velocity and $U_{\infty}$ is the free stream velocity. Thus, the wall shear stress

$$
\tau_{w}=\rho v_{*}^{2}=-\mu \frac{d v_{*}}{d x} G(\xi)
$$

where $\quad G(\xi)=\int_{0}^{y_{o}^{+}}\left(u^{+}\right)^{2} d y^{+}$.

Substituting $v_{*}=U_{\infty} / \xi$ in Eq.(3.4)

$$
\frac{U_{\infty}}{v} d x=d\left(\frac{U_{\infty} x}{v}\right)=d\left(\operatorname{Re}_{x}\right)=G(\xi) d \xi
$$
obtained

Upon integrating Eq.(3.5), the local Reynolds number based on the downstream distance $x$ is

$$
\operatorname{Re}_{x}=\frac{U_{\infty} x}{\nu}=\int_{0}^{\xi} G(\xi) d \xi
$$

$G(\xi)$ obtained from Spalding's formulation has the form

$$
G(\xi)=\frac{\xi^{3}}{3}+\frac{A}{\kappa^{2}}\left[e^{z}\left(z^{2}-2 z+2\right)-2-\frac{z^{3}}{3}-\frac{z^{4}}{4}-\frac{z^{5}}{10}-\frac{z^{6}}{36}\right]
$$

where $z=\kappa \xi$. Consequently $\operatorname{Re}_{x}$ will be

$$
\operatorname{Re}_{x}=\frac{\xi^{4}}{12}+\frac{A}{\kappa^{3}}\left[e^{z}\left(z^{2}-4 z+6\right)-6-2 z-\frac{z^{4}}{12}-\frac{z^{5}}{20}-\frac{z^{6}}{60}-\frac{z^{7}}{252}\right]
$$

$G(\xi)$ can be approximated explicitly by an exponential curve fit for $20 \leq \xi \leq 40$

$$
G(\xi)=8.409 \exp (0.475 \xi)
$$

$G(\xi)$ is plotted as a function of $\xi$ in Fig.6. In the same figure $G(\xi)$ obtained from the logarithmic law is also plotted. 


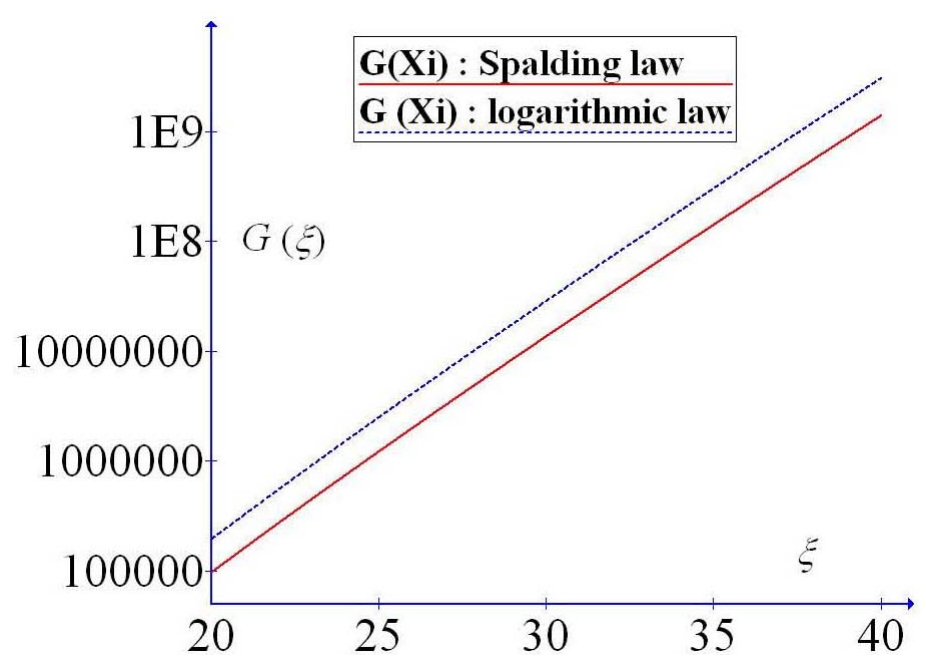

Fig.6. $G(\xi)$ as function $\xi$.

Combining Eqs (3.6) and (3.9)

$$
\operatorname{Re}_{x}=17.703 \exp (0.475 \xi)
$$

and consequently, the coefficient of local skin friction

$$
C_{f}=\frac{\tau_{w}}{\frac{1}{2} \rho U_{\infty}^{2}}=\frac{2}{\xi^{2}}=\frac{0.451}{\ln ^{2} 0.0565 \mathrm{Re}_{x}} .
$$

The measurements which are going to be used for comparison with Eq.(3.10) were performed by Österlund (1999), Österlund et al. (2000); Österlund and Nagib (2000) etc.. Figure 7 shows the comparison. It is observed that Eq.(3.10) overestimates the skin friction by approximately 9\%. Hence, likewise Prandtl's adjustment of theoretical Eq.(2.8), Eq.(3.10) is adjusted to give a better agreement with Österlund (1999) data. A multiplying factor of 0.9179 is introduced to Eq.(3.10) to predict the data with a high degree of accuracy (coefficient of determination, $R^{2}=0.997$ ). The adjusted equation is given below

$$
C_{f}=\frac{2}{\xi^{2}}=\frac{0.414}{\ln ^{2} 0.0565 \mathrm{Re}_{x}}
$$

Equation (3.11) is shown in Fig.7. A similar calculation is made using the logarithmic law and the result is also shown in Fig.7. It is observed that the calculation of $C_{f}\left(\operatorname{Re}_{x}\right)$ based on the logarithmic law overestimates the skin friction by about $23 \%$. The authors can offer an explanation for this. It is revealed from Fig. 6 that the value of $G(\xi)$ obtained from the logarithmic law is higher than that obtained from Spalding's law. Consequently, for the same value of $\mathrm{Re}_{x}$, the value of $\xi$ is lesser for the logarithmic law which leads to a higher value of the skin friction in the case of the logarithmic law. 


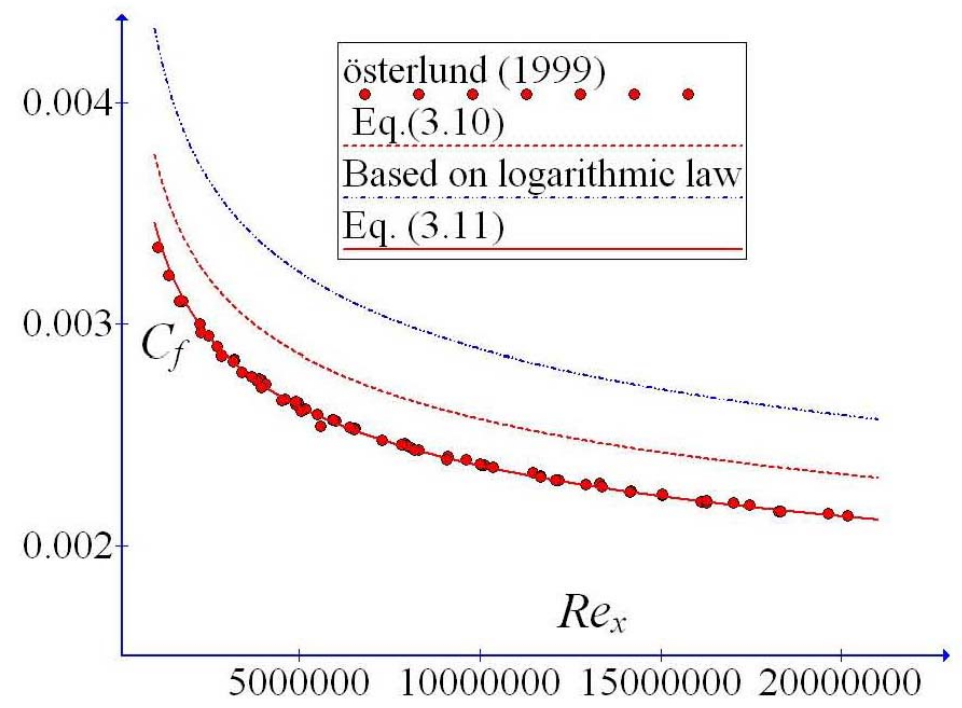

Fig.7. Theoretical $C_{f}\left(\operatorname{Re}_{x}\right)$ relation compared with Österlund (1999).

Clauser (1956) pointed out that "In almost no case does the turbulent boundary layer have a reliable point of origin from which $x$ may be measured. Certainly, experimental data can be interpreted with more generality and reliability on the basis of the thickness Reynolds numbers rather than with $x$-Reynolds numbers".

In view of the above, an attempt has been made to establish the relation between the skin friction, $C_{f}$ and the momentum thickness Reynolds number, $\operatorname{Re}_{\theta}\left(=U_{o} \theta / v\right)$. The momentum thickness $\theta$ is also a measure of boundary layer thickness. It has the form

$$
\theta=\int_{0}^{\infty} \frac{u}{U_{\infty}}\left(1-\frac{u}{U_{\infty}}\right)=\frac{v}{v_{*}} \int_{0}^{y_{o}^{+}} \frac{u^{+}}{\xi}\left(1-\frac{u^{+}}{\xi}\right) d y^{+},
$$

and consequently the momentum thickness Reynolds number

$$
\operatorname{Re}_{\theta}=\frac{U_{\infty} \theta}{v}=\int_{0}^{y_{o}^{+}} u^{+}\left(1-\frac{u^{+}}{\xi}\right) d y^{+} .
$$

The momentum integral relation for a flat plate (zero pressure gradients) is

$$
C_{f}=\frac{2}{\xi^{2}}=2 \frac{d \mathrm{Re}_{\theta}}{d \mathrm{Re}_{x}}
$$

Consequently,

$$
\operatorname{Re}_{\theta}=\int_{0}^{\xi} \frac{1}{\xi^{2}} G(\xi) d \xi
$$

From Eq.(3.7), $\frac{1}{\xi^{2}} G(\xi)$ can be approximated as 


$$
\frac{1}{\xi^{2}} G(\xi)=0.083 \exp (0.404 \xi)
$$

Introducing this approximation into Eq.(3.13) one obtains

$$
\operatorname{Re}_{\theta}=0.205 \exp (0.404 \xi)
$$

Rearranging the above equation

$$
C_{f}=\frac{2}{\xi^{2}}=\frac{0.326}{\ln ^{2} 4.878 \mathrm{Re}_{\theta}} .
$$

Equation (3.15) is compared with the experimental data of Österlund's (1999) in Fig.8. It is revealed that Eq.(3.15) overestimates the skin friction by approximately $9 \%$. Hence, Eq.(3.15) is adjusted to give a better agreement with Österlund's (1999) measurements. Multiplying Eq.(3.15) by a factor of 0.9110 gives an exceptionally good fit to the data with the coefficient of determination, $R^{2}=0.999$. The adjusted equation is given below

$$
C_{f}=\frac{0.297}{\ln ^{2} 4.878 \mathrm{Re}_{\theta}}
$$

Equation (3.16) is plotted in Fig.8. In a similar way $C_{f}\left(\mathrm{Re}_{\theta}\right)$ is determined using the logarithmic law and the result is also shown in Fig.8. Computation based on the logarithmic law overestimates the skin friction by about $23 \%$. The cause of deviation of the theoretical value of $C_{f}\left(\operatorname{Re}_{\theta}\right)$ is the same as explained before in the case of $C_{f}\left(\operatorname{Re}_{x}\right)$.

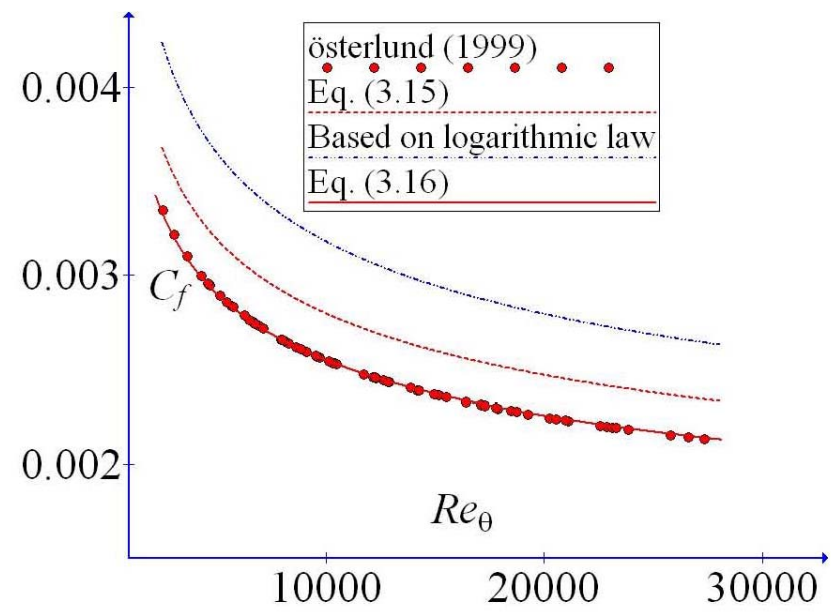

Fig.8. Theoretical $C_{f}\left(\operatorname{Re}_{\theta}\right)$ relation compared with Österlund (1999).

Distribution of shear stresses is obtained combining Eqs (3.2) and (3.4) 


$$
\tau=\tau_{w}\left[1-\frac{G\left(u^{+}\right)}{G(\xi)}\right]
$$

Subtracting $\tau_{l}=\tau_{w} / f^{\prime}\left(u^{+}\right)$from Eq.(3.17)

$$
\frac{\tau_{t}}{\tau_{w}}=1-\frac{G\left(u^{+}\right)}{G(\xi)}-\frac{1}{f^{\prime}\left(u^{+}\right)}
$$

$\tau_{t} / \tau_{w}$ has been computed from Eq.(3.18) using Spalding's formulation for the law of the wall and results are compared with Smith's (1994) data in Fig.9. The result is very encouraging.

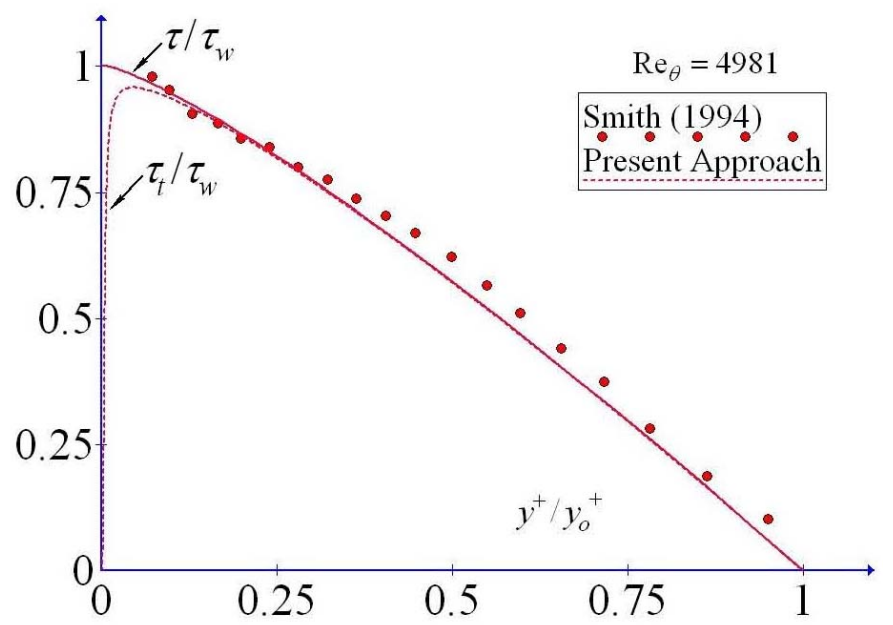

Fig.9. Computed shear stress for zero pressure gradient flow compared with Smith (1994).

\section{Conclusion}

Computations of pipe flow and flat plate resistances have been made using Spalding's law of the wall. This law is considered valid throughout the boundary layer. Results show that smooth pipe flow problems can be successfully solved through this law of the wall approach. Calculation of the local skin friction for a flat plate shows approximately $9 \%$ overestimation. So, necessary adjustment has been made for a better approximation. The proposed Eqs (3.11) and (3.16) are very simple and capable to approximate skin friction with high degree of accuracy.

A comparison between computed values of shear stress for both the cases of flow reveals that the law of the wall approach can also give a clue as to the stress field.

\section{Nomenclature}

$A$ - cross sectional area of the pipe and constant appearing in Spalding's law

$B$ - constant appearing in the logarithmic law

$C_{f}$ - coefficient of skin friction, $2 / \xi^{2}$

$D_{H} \quad$ - hydraulic diameter 


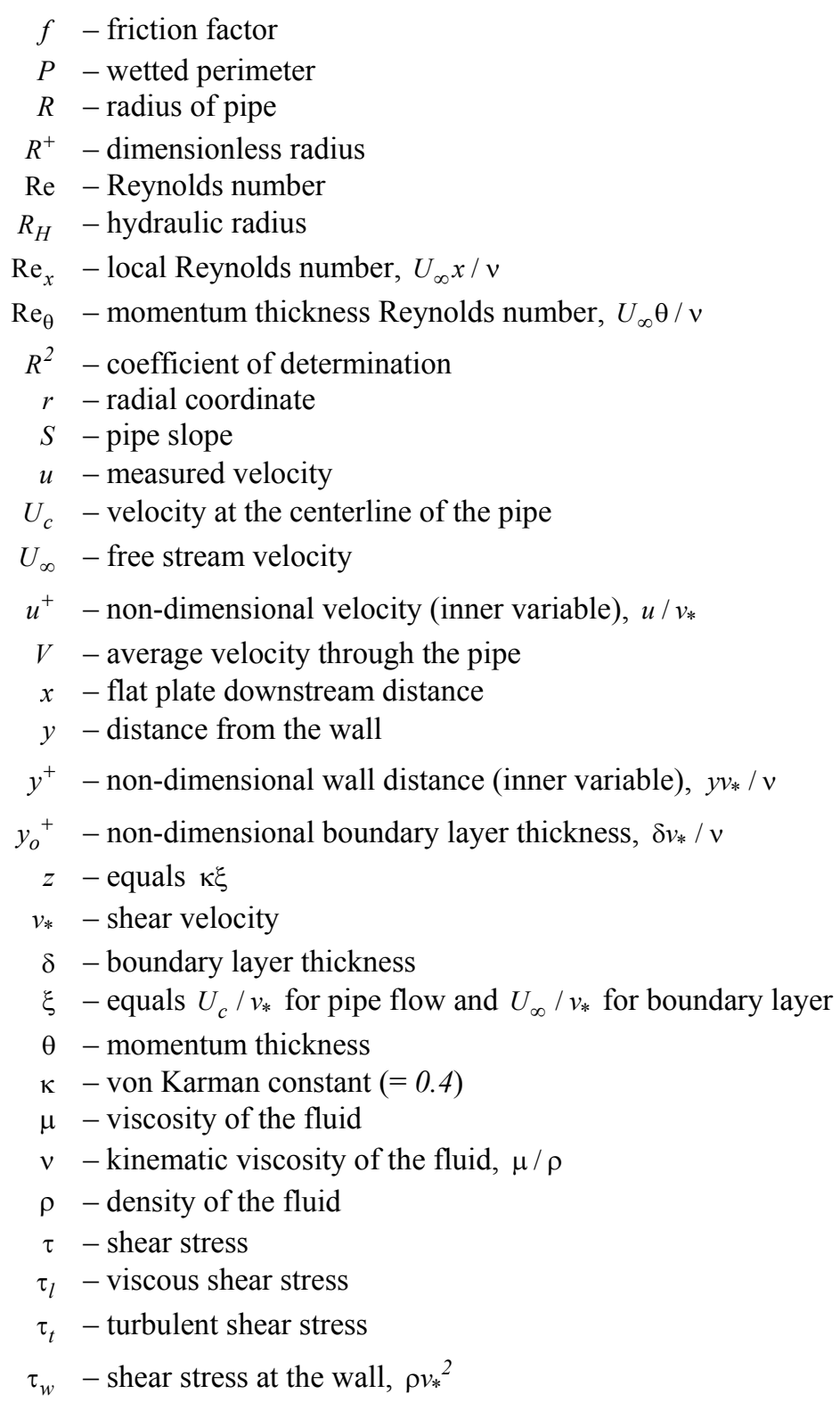

\section{References}

Clauser F. H. (1956): The turbulent boundary layer. - Adv. In Appl. Mech., vol.4, pp.1-51.

Kestin J. and Persen L.N. (1962): The transfer of heat across a turbulent boundary layer at very high Prandtl Numbers. - Int. J. Heat Mass Transfer, vol.5, pp.355-371.

Laufer J. (1954): The structure of turbulence in fully developed pipe flow. - Report 1174, NACA.

McKeon B.J., Swanson C.J., Zagarola M.V., Donnelly R.J. and Smits A.J. (2004): Friction factor for smooth pipe flow. - J. Fluid Mech., vol.511, pp.41-44.

Nikuradse J. (1932): Gesetzmässikeit der turbulenten strömug in glatten Rohren. - Forschg. Arb. Ing. Wes., 356.

Österlund J.M. (1999): Experimental Studies of zero pressure - gradient turbulent boundary layer flow. - Ph. D. Thesis, Royal Institute of Technology, Stockholm, Sweden. 
Österlund J.M., Johansson A.V., Nagib H.M. and Hites M.H. (2000): A note on the overlap region in turbulent boundary layers. - Phys. Fluids, vol.12, No.1, pp.1-4.

Österlund J.M. and Nagib H.M. (2000): Comment on "A note on the overlap region in turbulent boundary layers. Phys. Fluids, vol.12, No.9, pp.2360-2363.

Prandtl L. (1933): Neuere ergebnisse der turbulenzforschung. - Z. VDI. 77, Nr. 5, pp.105-114.

Prandtl L. (1935): The mechanics of viscous fluids. - In: W. F. Durand. Aerodynamics Theory, III.

Smith R.W. (1994): The effect of Reynolds number on the structure of turbulent boundary layers. - Ph. D. Thesis Department of Mechanical and Aerospace Engineering, Princeton University.

Spalding D.B. (1961): A single formula for the law of wall. - J. Appl. Mech., vol.28, Ser. E, pp.455-458.

Swanson C.J., Julian B., Ihas G.G. and Donnelly R.J. (2002): Pipe flow measurements over a wide range of Reynolds numbers using liquid helium and various gases. - J. Fluid Mech., vol.461, pp.51-60.

Received: November 24, 2014

Revised: October 5, 2015 